\title{
Critical Process Parameters Evaluation of Modified Nanoprecipitation Method on Lomustine Nanoparticles and Cytostatic Activity Study on L132 Human Cancer Cell Line
}

\author{
Archana Mehrotra' and Jayanta K. Pandit ${ }^{2 *}$
}

${ }^{1}$ Oriental College of Pharmacy and Research, Oriental University, Sanwer Road, Indore, M.P, India

${ }^{2}$ Department of Pharmaceutics, Institute of Technology, Banaras Hindu University, Varanasi- 221005, U.P, India

\begin{abstract}
This work was focused on identification and evaluation of process parameters of modified nanoprecipitation method, for fabrication of lomustine nanoparticles, with the aim of reducing cancer cell viability at low concentration of lomustine. The parameters controlling particle size, mostly in nanosize, were solvent/nonsolvent composition and emulsification speed of homogenizer along with aqueous phase volume. This controlled particle size is below $250 \mathrm{~nm}$. The stabilizer concentration controlled particle size is within $68 \mathrm{~nm} \pm 0.89$ to $137 \pm 0.94 \mathrm{~nm}$ with PDI $0.06 \pm 0.008$ to $0.25 \pm 0.001$. But, the stabilizer addition mode showed more uniform size distribution with PDI $0.085 \pm 0.004$. Entrapment efficiency was maintained well above $47 \pm 0.23 \%$. The drug release pattern was monophasic with controlled release over $24 \mathrm{hrs}$. In the method used, drug content was affected by ratio of polymer to drug to organic solvent, as well as homogenization speed and time. Percentage viable cells of L132 human lung cancer cell line remained, were only $5 \%$ at $100 \mu \mathrm{g} / \mathrm{ml}$ lomustine equivalent PLA nanoparticles.
\end{abstract}

Keywords: Modified nanoprecipitation; Human lung cancer cell line 132; Stabilizer PVA; PLA nanoparticles; Homogenization

\section{Introduction}

Biocompatible nanoparticles as drug delivery vehicles provide several advantages, including protection of the encapsulated pharmaceutical substance, improved efficacy, fewer adverse effects, controlled release and drug targeting [1]. In the nanoparticle formulation, particular interest has been focused on the use of polyesters materials such as poly (d,l-lactide) (PLA) and poly(d,llactide-co-glycolide) (PLGA), which undergo scission in the body to monomeric units of lactic acid, as a natural intermediate in carbohydrate metabolism. The degradation rate is dependent on several parameters, such as crystallinity (crystalline PLA degrades slower), MW (low MW PLAs degrade faster) environment $(\mathrm{pH}$, ionic strength, temperature) and particle morphology (porous particles degrade faster) [2,3]. At neutral $\mathrm{pH}$ (in vitro liquid environment without enzymes), PLA nanoparticles remain relatively stable over tens of days [3]. Owing to their biocompatibility and biodegradability properties, nanoparticles of these polymers are investigated for wide applications, using several preparation procedures.

Some of the techniques commonly used include nanoprecipitation/ solvent displacement procedures [4], salting-out [5], emulsification solvent diffusion [6], and solvent evaporation. In order to produce small and low polydisperse nanoparticle population, the nanoprecipitation method is one of the easiest. In the nanoprecipitation process, particle formation is spontaneous, because the polymer precipitates in the aqueous environment. The Marangoni effect is considered to explain the process [7]: solvent flow, diffusion and surface tension at the interface of the organic solvent and the aqueous phase cause turbulences, which form small droplets containing the polymer. Subsequently, as the solvent diffuses out from the droplets, the polymer precipitates. Finally, the organic solvent is typically evaporated with the help of a vacuum. No emulsification step (which is usually part of a nanoparticle preparation process), laborious processing conditions or special laboratory ware is needed. The size of the nanoparticles prepared by nanoprecipitation varies typically from 100 to $500 \mathrm{~nm}$.
Previously, in nanoprecipitation method, a single step was involved in the formation of nanoparticles (NP). On addition of organic phase containing drug and polymer into aqueous phase, desolvation of polymer occured rapidly due to diffusion of solvent into aqueous phase. This resulted into precipitation of polymer along with drug entrapped into the polymer matrix, to give instantaneous formation of NP with drug distributed into the polymer matrix [8]. The nanoprecipitation technique was relatively straightforward and rapid, and offered reproducible particle size with a narrow distribution [9].

Presently in modified nanoprecipitation method, we tried to put up a concept of increasing surface energy of the drug in the formulation, which ultimately gets partitioned in favour of the bulk, leading to negative adsorption and zero order drug release system. Pseudoplastic flow of polymeric solution of formulation begin to align at long axes in the direction of flow, after applying shear stress and results in effective lowering of size of dispersed particles. In the present study, to get more uniform size distribution and increased encapsulation efficiency of nanoparticles of lomustine anticancer drug, with increasing anticancer activity at lower dose of lomustine equivalent, the nanoparticles were fabricated by modifying the nanoprecipitation method with homogenization. The different process parameters like polymer concentration, drug concentration, stabilizer concentration, stabilizer incorporation mode, aqueous phase volume, addition of nonsolvent

${ }^{*}$ Corresponding author: Prof. J. K. Pandit, Department of Pharmaceutics, Institute of Technology, Banaras Hindu University, Varanasi- 221005, U.P, India, Tel: +919451570863; E-mail: jkpandit@bhu.ac.in

Received July 13, 2012; Accepted September 24, 2012; Published September 26, 2012

Citation: Mehrotra A, Pandit JK (2012) Critical Process Parameters Evaluation of Modified Nanoprecipitation Method on Lomustine Nanoparticles and Cytostatic Activity Study on L132 Human Cancer Cell Line. J Nanomed Nanotechol 3:149. doi:10.4172/2157-7439.1000149

Copyright: (c) 2012 Mehrotra A, et al. This is an open-access article distributed under the terms of the Creative Commons Attribution License, which permits unrestricted use, distribution, and reproduction in any medium, provided the original author and source are credited. 
ethanol as a cosolvent, homogenization speed and time were evaluated affecting the particle size, encapsulation efficiency and in vitro drug release.

\section{Materials and Methods}

Polymer PLA was a gift sample from Purac Biomaterials, Gorinchem, The Netherlands. Lomustine pharmaceutical grade was obtained from Fujian Provincial Medicines and Health Products, Xiamen Import and Export Corporation (China). Acetone (Qualigens, Mumbai, India), PVA (CDH, New Delhi, India) and distilled water were used throughout the study. Homogenizer was used of Ika Laborthechnik, Germany.

\section{Preparation of nanoparticles}

Nanoparticles were prepared by the nanoprecipitaion method specified by Wu et al. [10]. For basic formulation, (Batch APL) PLA and lomustine were dissolved in acetone, and was homogenized with homogenizer (Ika Labortechnik) at 19,000 rpm for $5 \mathrm{~min}$. This solution was then added dropwise to $1 \% \mathrm{w} / \mathrm{v}$ aqueous polyvinyl alcohol (PVA) solution, by homogenization for $20 \mathrm{~min}$. Then, organic solvent was removed using rotary evaporator (B-480 Buchi, Switzerland). The final volume of suspension was adjusted to $10 \mathrm{ml}$, with deionized water. Nanoparticles were separated by centrifugation at $19,000 \mathrm{rpm}$ for 30 min, and redispersed in deionized water. Placebo nanoparticles were prepared as per above method omitting the drug. Table 1 shows the formulae for the fabrication of the drug loaded nanoparticles (DNPs).

In each set of experiments, only one formulation variable was changed at a time while the other parameters were kept same, as those described for the basic formulation (formulation batch APL). The following protocol modifications were used to study the effect of process and preparative variables for respective batches.

1. Variable polymer PLA concentration in batch APL1 and APL2.

2. Variable drug concentration in batch APL3 and APL4.

3. Increasing aqueous phase volume to $100 \mathrm{ml}$, instead of $50 \mathrm{ml}$ in batch APL5.

4. Increasing stabilizer PVA concentration in batch APL6 and APL7.

\section{Ethanol Particle Size PDI *Drug Encapsulation (nm) (Mean \pm content $(\mathrm{mg})$ efficiency $(\text { Mean } \pm \text { SD })^{*}$ SD $)^{*}$ (Mean \pm SD)}

\begin{tabular}{|c|c|c|c|c|c|}
\hline 1. & APL & 200 & 10 & 20 & $1 \% \mathrm{w} / \mathrm{v}$ \\
\hline 2. & APL1 & 100 & 10 & 20 & $1 \% w / v$ \\
\hline 3. & APL2 & 300 & 10 & 20 & $1 \% w / v$ \\
\hline 4. & APL3 & 200 & 20 & 20 & $1 \% w / v$ \\
\hline 5. & APL4 & 200 & 30 & 20 & $1 \% w / v$ \\
\hline 6. & APL5 & 200 & 10 & 20 & $100 \mathrm{ml} 1 \% \mathrm{w} / \mathrm{v}$ \\
\hline 7. & APL6 & 200 & 10 & 20 & $2 \% w / v$ \\
\hline 8. & APL7 & 200 & 10 & 20 & $3 \% \mathrm{w} / \mathrm{v}$ \\
\hline 9. & APL8 & 200 & 10 & 20 & $1 \% w / v$ \\
\hline 10. & APL9 & 200 & 10 & 20 & $1 \% w / v$ \\
\hline 11. & APL10 & 200 & 10 & 20 & $1 \% w / v$ \\
\hline 12. & APL11 & 200 & 10 & 20 & $1 \% \mathrm{w} / \mathrm{v}$ \\
\hline 13. & APL12 & 200 & 10 & 20 & $1 \% w / v$ \\
\hline 14. & APL13 & 200 & 10 & 20 & $1 \% w / v$ \\
\hline 15. & APL14 & 200 & 10 & 20 & $1 \% w / v$ \\
\hline 16. & APL15 & 200 & 10 & 20 & $1 \% \mathrm{w} / \mathrm{v}$ \\
\hline 17. & APL16 & 200 & 10 & 20 & $1 \% w / v$ \\
\hline 18. & APL17 & 200 & 10 & 20 & $1 \% \mathrm{w} / \mathrm{v}$ \\
\hline 19. & APL18 & 200 & 10 & 20 & $1 \% \mathrm{w} / \mathrm{v}$ \\
\hline 20. & APL19 & 200 & 10 & 20 & $1 \% w / v$ \\
\hline
\end{tabular}

\begin{tabular}{|c|c|c|c|}
\hline $115 \pm 1.2$ & $\begin{array}{l}0.13 \pm \\
0.001\end{array}$ & 7.9 & $79 \pm 0.18 \%$ \\
\hline $68 \pm 0.89$ & $\begin{array}{l}0.06 \pm \\
0.008\end{array}$ & 4.7 & $47 \pm 0.23 \%$ \\
\hline $137 \pm 0.94$ & $\begin{array}{l}0.18 \pm \\
0.004\end{array}$ & 9.0 & $90 \pm 0.34 \%$ \\
\hline $122 \pm 1.6$ & $\begin{array}{l}0.22 \pm \\
0.003\end{array}$ & 13.8 & $69 \pm 0.07 \%$ \\
\hline $128 \pm 0.75$ & $\begin{array}{l}0.25 \pm \\
0.001\end{array}$ & 21.9 & $73 \pm 0.14 \%$ \\
\hline $117 \pm 0.63$ & $\begin{array}{l}0.1 \pm \\
0.001\end{array}$ & 8.5 & $85 \pm 0.37 \%$ \\
\hline $125 \pm 0.92$ & $\begin{array}{l}0.086 \pm \\
0.017\end{array}$ & 8.3 & $83 \pm 0.08 \%$ \\
\hline $130 \pm 0.78$ & $\begin{array}{l}0.092 \pm \\
0.006\end{array}$ & 8.8 & $88 \pm 0.15 \%$ \\
\hline $136 \pm 0.99$ & $\begin{array}{l}0.085 \pm \\
0.004\end{array}$ & 8.1 & $81 \pm 0.06 \%$ \\
\hline Unstable & - & - & - \\
\hline $123 \pm 0.76$ & $\begin{array}{l}0.074 \pm \\
0.007\end{array}$ & 8.4 & $84 \pm 0.19 \%$ \\
\hline $117 \pm 1.1$ & $\begin{array}{l}0.079 \pm \\
0.004\end{array}$ & 8.8 & $88 \pm 0.24 \%$ \\
\hline $75 \pm 1.6$ & $\begin{array}{l}0.083 \pm \\
0.002\end{array}$ & 9.3 & $93 \pm 0.17 \%$ \\
\hline $133 \pm 1.4$ & $\begin{array}{l}0.081 \pm \\
0.016\end{array}$ & 8.6 & $86 \pm 0.26 \%$ \\
\hline $110 \pm 0.83$ & $\begin{array}{l}0.088 \pm \\
0.003\end{array}$ & 9.5 & $95 \pm 0.31 \%$ \\
\hline $844 \pm 1.6$ & $\begin{array}{l}0.30 \pm \\
0.018\end{array}$ & 5.5 & $55 \pm 0.19 \%$ \\
\hline $799 \pm 1.8$ & $\begin{array}{l}0.27 \pm \\
0.012\end{array}$ & 6.2 & $62 \pm 0.26 \%$ \\
\hline $437 \pm 0.87$ & $\begin{array}{l}0.23 \pm \\
0.001\end{array}$ & 6.6 & $66 \pm 0.23 \%$ \\
\hline $238 \pm 0.65$ & $\begin{array}{l}0.19 \pm \\
0.004\end{array}$ & 7.2 & $72 \pm 0.34 \%$ \\
\hline $100 \pm 1.0$ & $\begin{array}{l}0.066 \pm \\
0.003\end{array}$ & 8.9 & $89 \pm 0.25 \%$ \\
\hline
\end{tabular}


5. Addition of nonsolvent ethanol of drug, as a cosolvent to acetone in batches APL10, APL11 and APL12.

6. In formulations APL8 and APL9, effect of stabilizer PVA incorporation mode was studied: a) Incorporation into the aqueous phase, prior to phase mixing (Batch APL) b) Incorporation into the organic phase, prior to phase mixing (Batch APL8) and c) Immediately after phase mixing (Batch APL9).

7. In another set of experiments, the organic solvents evaporation rate modified by applying one of the following evaporation conditions, for respective formulations APL13 and APL14. a) At atmospheric pressure at room temperature (Batch APL). b) Pressure reduced gradually from 180 to $40 \mathrm{mmHg}$ at $35^{\circ} \mathrm{C}$ (Batch APL13) and c) Pressure reduced instantaneously to 40 $\mathrm{mmHg}$ at $35^{\circ} \mathrm{C}$ (Batch APL14).

8. The effect of increased homogenization speed at different time was examined for respective formulations. a) at 10,000 rpm for 5 min.(Batch APL15 ) b) at 19,000 rpm for 5 min.(Batch APL16) c) at 21,000 rpm for $5 \mathrm{~min}$.(Batch APL17) d) at 10,000 rpm for $20 \mathrm{~min}$.(Batch APL18) e) at 19,000 rpm for $20 \mathrm{~min}$. (Batch APL) f) at 21,000 rpm for $20 \mathrm{~min}$.(Batch APL19)

\section{Nanoparticle characterization}

Particle size, polydispersity and morphology: Nanoparticle mean diameter and polydispersity index were determined using photon correlation spectroscopy (PCS) (Zetasizer Malvern instruments DTS Ver 4.10). The analysis was performed at a scattering angle $90^{\circ}$ and at a temperature of $25^{\circ} \mathrm{C}$, using samples appropriately diluted with ultrapurified water. Surface morphology was observed by Scanning Electron Microscopy (SEM), for which a thin film of aqueous dispersion of nanoparticles was applied on double stick tape, over an aluminium stub and air dried, to get uniform layer of particles. These particles were coated with gold using sputter gold coater, and subjected to SEM on Leo 435 VP, Cambridge, UK.

Drug content and encapsulation efficiency: The drug content in nanoparticles was determined spectrophotometrically. Drug loaded nanoparticles dispersed in deionized water, were centrifuged at 19,000 rpm for $30 \mathrm{~min}$ and discarded the supernatant. The residue obtained was washed twice with water and a $0.50 \mathrm{ml}$ aliquot of nanoparticles aqueous suspension was evaporated to dryness in a petri dish at $35^{\circ} \mathrm{C}$, and residue dissolved in acetone $(1 \mathrm{ml})$, a common solvent for PLA and lomustine. Then, it was diluted with ethanol $95 \%$ and followed by estimation of the drug in triplicate, using UV spectrophotometer at 230 $\mathrm{nm}$. The drug content was calculated using following equation.

$$
\text { Drug content }=\frac{\text { Mass of drug in nanoparticles }}{\text { Mass of nanoparticles re covered }} \times 100
$$

The encapsulation efficiency was calculated as following equation.

$$
\% \text { Encapsulation efficiency }=\frac{\text { Mass of drug in nanoparticles }}{\text { Mass of drug used in formulation }} \times 100
$$

In-vitro drug release studies: Lomustine release from different batches of nanoparticles was determined using dialysis-bag diffusion technique, under magnetic stirring. $25 \mathrm{mg}$ DNPs were redispersed in $3 \mathrm{ml}$ phosphate buffer saline solution, $\mathrm{pH} 7.4$ and placed in a dialysis membrane bag (cellulose membrane, molecular weight cutoff 12,000), hermetically closed, and immersed into $50 \mathrm{ml}$ of PhosphateBuffered Saline (PBS), $\mathrm{pH}$ 7.4. The entire system was kept at $37^{\circ} \mathrm{C}$ with continuous magnetic stirring. At appropriate time intervals, $3 \mathrm{ml}$ of release medium was removed and the same volume of fresh PBS solution was added into the system. The amount of lomustine in the release medium was evaluated by UV spectrophotometer (Elico SL164), at $\lambda_{\max } 230.4 \mathrm{~nm}$ by dilution with ethanol $95 \% \mathrm{w} / \mathrm{v}$.

For evaluation of release kinetics, the obtained release data were fitted into first order, zero order and Higuchi equations. Selection of the best model was based on the comparisons of the relevant correlation coefficients.

\section{In-vitro anticancer activity test}

The antiproliferative action of lomustine and the nanoparticle formulation (Batch APL) were tested on human lung cancer cell line L132. L132 cells were seeded at a density of $1 \times 10^{4}$ cells/well in $180 \mu \mathrm{l}$ growth medium, RPMI-1640 in 96-well plates and incubated for $24 \mathrm{~h}$, prior to the addition of $20 \mu \mathrm{l}$ solutions of lomustine, and batch APL nanoparticles with final concentrations of $20,50,75$ and $100 \mu \mathrm{g} / \mathrm{ml}$ Cells were incubated for $24 \mathrm{~h}$, before the addition of the MTT $(20 \mu \mathrm{l} /$ well at a concentration of $5 \mathrm{mg} / \mathrm{ml}$ in PBS buffer). After further incubation of $4 \mathrm{~h}$, the media was removed and replaced with $150 \mu \mathrm{LMSO}$. The absorbance was read at $570 \mathrm{~nm}$ in a microplate reader, following a 300 -second automixing. The cell viability (\%) was calculated according to the following equation: Cell viability $(\%)=($ OD570 (sample)/OD570 $($ control) $) \times 100$, where OD570 (sample) represents measurement from the wells treated with samples, and OD570 (control) from the wells treated with RPMI 1640 media only.

\section{Statistical analysis}

Results are given as mean \pm SD. Mean values of nanoparticle size, polydispersity index, and encapsulation efficiency were compared using the Student's t-test. Differences are considered significant, at a level of $\mathrm{p}<0.05$. In vitro data were analyzed with a one-way ANOVA. Critical value of $\mathrm{F}$ was considered at $5 \%$ level of significance.

\section{Results}

\section{Particle size and polydispersity index}

As shown in Table 1, the mean particle size and polydispersity index (PDI) of the lomustine nanoparticles were less than $250 \mathrm{~nm}$ and 0.3 , respectively, except for nanoparticles prepared by homogenization at different speeds for $5 \mathrm{~min}$ (size $844 \pm 1.6 \mathrm{~nm}$ to $437 \pm 0.87 \mathrm{~nm}$, for homogenization at $10,000 \mathrm{rpm}$ to $21,000 \mathrm{rpm}$ for $5 \mathrm{~min}$, for batch APL15-17).

PDI: Polydispersity Index; SD: Standard Deviation for three determinations.

Batch APL9 where PVA was incorporated after organic and aqueous phase mixing, resulted in unstable formulation showing compact mass.

${ }^{*}$ Drug content: Indicates actual loading of drug in nanoparticles i.e. amount of drug present in the recovered nanoparticles, in formulation. In the method used, it is affected by ratio of polymer to drug to organic solvent (APL1, APL3, APL4), as well as homogenization speed and time (APL15, APL16, APL17)

The results showed in Table 1 indicated that the small mean size and PDI value depended on the increasing homogenization speed, non-solvent (ethanol) addition, and instantaneous organic solvent evaporation rate (Batch APL14). The 20 min homogenization was necessary, to get the nanoparticles of size less than $250 \mathrm{~nm}$ (Batch APL18-APL19). Increasing polymer, drug and stabilizer PVA 
concentration affected the particle size and PDI significantly $(\mathrm{p}<0.05)$, producing particles of size of $68 \mathrm{~nm} \pm 0.89$ to $137 \pm 0.94 \mathrm{~nm}$ with PDI $0.06 \pm 0.008$ to $0.25 \pm 0.001$. PVA incorporated after the organic and aqueous phase mixing (Batch APL9) resulted in unstable formulation showing compact mass, therefore, not considered for characterization. PVA solution added in the organic solution (Batch APL8) showed increased particle size of $136 \mathrm{~nm} \pm 0.99$, with more uniform size distribution with PDI $0.085 \pm 0.004$

\section{Surface morphology}

The nanoparticles of basic formulation (Batch APL) observed by Scanning Electron Microscopy (SEM) were smooth and spherical in shape (Figure 1). Particle size determined by SEM correlated to that observed using Zeta Sizer.

\section{Encapsulation efficiency of nanoparticles}

The Encapsulation Efficiency (EE) of lomustine nanoparticles (Batch APL) was $79.0 \pm 0.18 \%$ (Table 1). EE was increased considerably, with increasing lomustine concentration (Batches APL3-4). EE was increased significantly $(\mathrm{p}<0.05)$ when acetone was evaporated instantaneously at $40 \mathrm{mmHg}$ with $95 \pm 0.31 \%$ (Batch APL14). It was increased only slightly $(\mathrm{p}>0.05)$ when stabilizer PVA was incorporated in organic phase, before mixing of two phases (Batch APL8 as compared to batch APL). In all other preparative parameters, tested EE was increased. EE of batches APL6 and APL10 were near about same along with particle size, but polydispersity was different, APL10 nanoparticles being more uniform in size than APL6.

\section{In vitro drug release}

Figure $2(\mathrm{~A}-\mathrm{H})$ shows the percentage release of lomustine from different batches (APL- APL19) in PBS ( $\mathrm{pH}$ 7.4). The nanoparticles initially showed slow controlled release. After 24 hours of dialysis in PBS ( $\mathrm{pH}$ 7.4), the percentages of lomustine released was $99 \%$ for batch APL with $\mathrm{t}_{75 \%} 9$ hours.

The PLA content affected the drug release kinetics. The increase in PLA content in the nanoparticles resulted in slower kinetics. The $\mathrm{t}_{75 \%}$ values in PBS ( $\mathrm{pH}$ 7.4) were about 5 hours, 9 hours, and 16 hours for batches APL1, APL and APL2, containing 100, 200, and $300 \mathrm{mg}$ of PLA, respectively (Figure 2A).

The effect of drug loading on lomustine release, was also studied. There was a difference in the release pattern of 20 and $30 \mathrm{mg}$ of lomustine-loaded nanoparticles. The $t_{75 \%}$ values for batches APL3 and APL4 were 13 and 15 hours, respectively in PBS ( $\mathrm{pH} 7.4$ ). Lomustine release was found to extend, with decrease in the drug loading (Figure 2B). Increasing the stabilizer PVA concentration (batches APL6-7, (Figure 2D) showed decreased drug release kinetics, with $\mathrm{t}_{75 \%}$ values 10 hours and 10.5 hours. Increasing the aqueous phase (PVA solution,

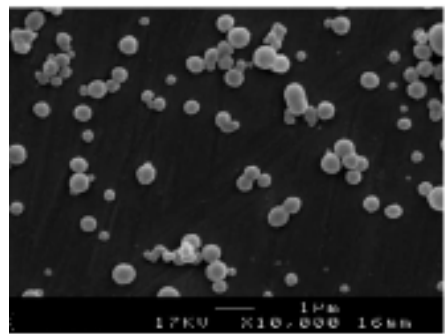

Figure 1: SEM Photograph of basic formulation nanoparticles (APL).
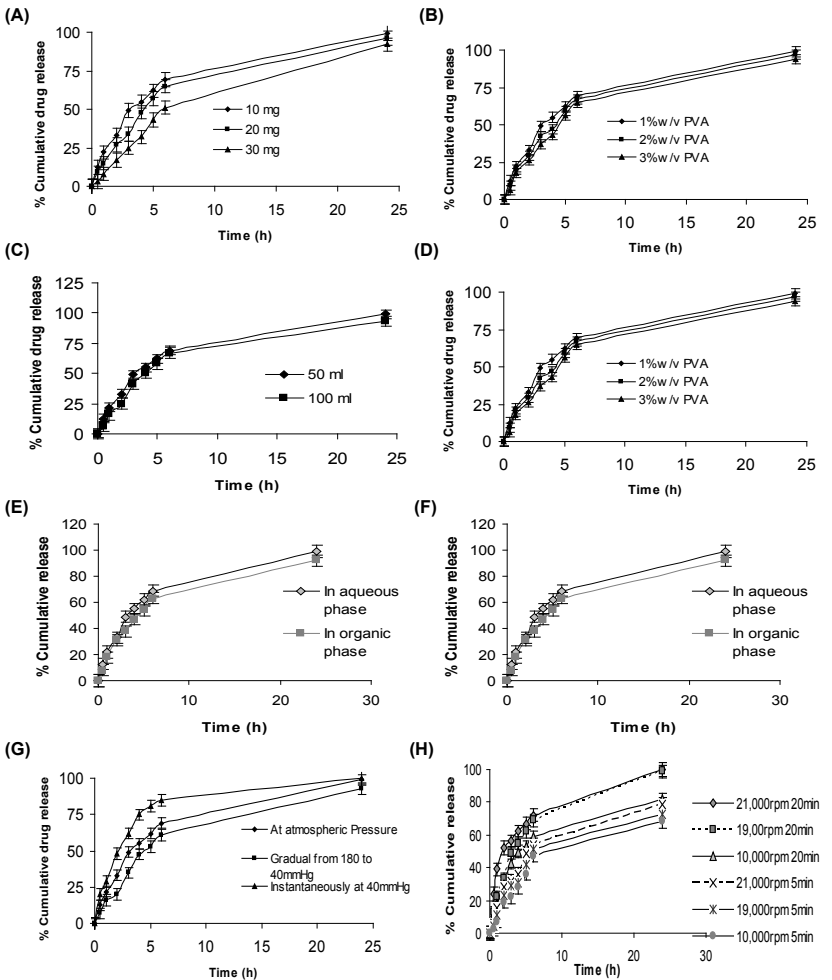

Bars represent $\pm \operatorname{SD}(n=3)$

Figure 2: In vitro drug release of lomustine as function of component variables. (A) Release of lomustine, batches APL and APL1, and APL2 as a function of PLA concentration. (B) Release of lomustine, batches APL, APL3, and APL4 as a function of lomustine concentration, (C) Release of lomustine, batches APL and APL5 as a function of aqueous phase (PVA solution) amount, (D) Release of lomustine, batches APL, APL6-7 as a function of PVA solution concentration, (E) Release of lomustine batches APL, APL8 as a function of PVA incorporation mode (F) Release of lomustine, batches APL10-12 as a function of co-solvent ethanol addition. (G) Release of lomustine, batches APL, APL13-14 as a function of organic solvent evaporation condition, $(\mathbf{H})$ Release of lomustine, batches APL, APL15-19 as a function of homogenization speed and time.

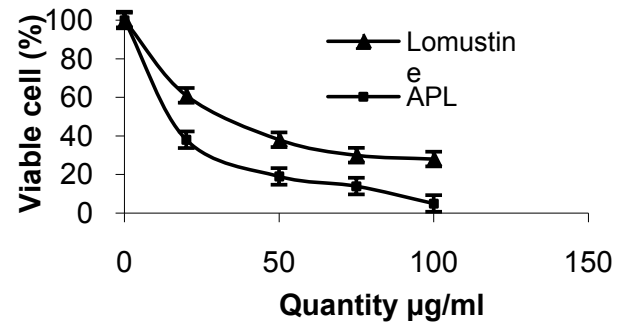

Figure 3: In vitro anticancer activity of lomustine and nanoparticles of basic formulation (Batch APL). Bars represent $\pm \operatorname{SD}(n=3)$.

batch APL5, (Figure 2C) volume slowed down the drug release to 93\% over 24 hours, with $t_{75 \circ}$ value 12.0 hours. Similar results were observed for the effect of PVA incorporation mode (Figure 2E). On addition of co-solvent ethanol (Batches APL10-12, (Figure 2F), drug release rate was increased showing $\mathrm{t}_{75 \%}$ values 8 hours, 6.5 hours and 4 hours, respectively. Lomustine release was increased to $100 \%$ and slowed to 93\%, after 24 hours with $\mathrm{t}_{75 \%}$ values 4 hours and 11 hours, when organic 
solvent was evaporated instantaneously and gradually (Batches APL14 and APL13, (Figure 2G), respectively.

There was significant effect of homogenization speed and time (Batches APL15-APL19, (Figure 2H) on lomustine release, showing $\mathrm{t}_{75 \%}$ values $>24,>24,21,18$ and 7 hours, respectively.

\section{Mechanism of drug release}

The release exponents ( $\mathrm{n})$ and correlation coefficients $\left(\mathrm{r}^{2}\right)$ of zero order equation are shown in Table 2 . The $\mathrm{r}^{2}$ values for a plot of zero order kinetics were more linear than for other plots. The values were in the range of 0.9498 to 0.9895 , which was always higher than other equations, indicating zero order kinetics of lomustine from the lomustine loaded nanoparticles. The release exponents (n) were $<1.0$, indicating non-fickian release mechanism.

\section{In-vitro anticancer activity}

In vitro anticancer activity was tested of lomustine, and nanoparticles batch APL (Figure 3).

As in figure percentage, viable cells were reduced to about $28 \%$ and $5 \%$ with $100 \mu \mathrm{g} / \mathrm{ml}$ lomustine, and nanoparticles batch APL, respectively. For 20,50 and $75 \mu \mathrm{g} / \mathrm{ml}$ lomustine concentration treatment, the viability of cancer cells treated by the nanoparticles batch APL was $38 \%, 19 \%$ and $14 \%$, respectively, in comparison with $61 \%, 38 \%$ and $30 \%$, for the cells treated with lomustine.

\section{Discussion}

In the present study, the nanoparticles were fabricated by modified nanoprecipitation method. The modified nanoparticle-production method, thus, achieved a synergistic effect of energy input during different phase mixing process, just in that short time period when nanodroplets were formed, organic solvent diffused, and nanoparticles solidified. Cavitation is believed to be the main cause of size reduction. Turbulence, collision and shear process are also responsible for size reduction [11].

\section{Particle size and polydispersity index}

The nanoprecipitation method is very sensitive to changes in composition [12,13]. The particle size depends on interplay between several variables, like the organic (polymer) phase viscosity, stabilizer(s), solvent properties and the polymer properties [13].

Previous studies identified polymer concentration, organic phase injection rate and needle gauge, as the principal size determinants of drug loaded nanoparticles, prepared by nanoprecipitation $[14,15]$. As shown by Chorny et al. [16] adjusting polymer concentration in the organic phase was found to be useful for the production of smaller sized nanoparticles, though restricted to a limited range of the polymer to drug ratio. Therefore, it was desirable to identify and characterize additional tools for nanoparticle size control

Lomustine remained in the inner organic phase, with the polymer. If the inner phase droplets in the outer phase represent lesser osmotic activity due to a water insoluble drug, flow of outer phase into the droplets does not occur [13]. Overall smaller sized particles obtained by this method, might be the outcome of concentration gradient and osmotic pressure, as well as shear stress.

The large sized nanoparticles were produced from a higher molecular weight polymer, also forming a more viscous organic solution [17] with increasing polymer concentration.

There was overlay of more PVA molecules on the surface of the droplets, involving an improvement in the protection of the droplets from coalescence, with increasing concentration of PVA. Also as described by Lamprecht et al. [18], this might be attributed to a reduction of the shear stress during the homogenization process, resulting from a higher viscosity of the aqueous phase and consequently, a less favorable mixing efficiency and larger emulsion droplets.

Ethanol was chosen to study the effect of a PLA non-solvent watermiscible organic phase component on the formulation properties. The incorporation of ethanol reduced the polylactide solubility in the organic solution, which in turn, caused an early precipitation of the polymer upon contact with the aqueous phase, and formation of

\begin{tabular}{|c|c|c|c|c|}
\hline S. No. & Formulations Batch Code & $t_{75 \%}$ & $\begin{array}{c}\text { Correlation coefficient }\left(r^{2}\right) \\
\text { Zero order }\end{array}$ & Release exponent (n) \\
\hline 1. & APL & 9 & 0.9794 & 0.51 \\
\hline 2. & APL1 & 5 & 0.9865 & 0.54 \\
\hline 3. & APL2 & 16 & $\begin{array}{l}0.9855 \\
0.9595\end{array}$ & 0.59 \\
\hline 4. & APL3 & 13 & 0.9629 & 0.53 \\
\hline 5. & APL4 & 15 & 0.9498 & 0.67 \\
\hline 6. & APL5 & 12 & $\begin{array}{l}0.9736 \\
0.9739\end{array}$ & 0.79 \\
\hline 7. & APL6 & 10 & $\begin{array}{l}0.9139 \\
0.9893\end{array}$ & 0.69 \\
\hline 8. & APL7 & 10.5 & - & 0.73 \\
\hline 9. & APL8 & 11.5 & 0.9690 & 0.71 \\
\hline 10. & APL9 & - & $\begin{array}{l}0.9895 \\
0.9892\end{array}$ & - \\
\hline 11. & APL10 & 8 & 0.9762 & 0.90 \\
\hline 12. & APL11 & 6.5 & 0.9824 & 0.51 \\
\hline 13. & APL12 & 4 & 0.9774 & 0.76 \\
\hline 14. & APL13 & 11 & $\begin{array}{l}0.9881 \\
0.9869\end{array}$ & 0.81 \\
\hline 15. & APL14 & 4 & 0.9891 & 0.84 \\
\hline 16. & APL15 & $>24$ & 0.9778 & 0.84 \\
\hline 17. & APL16 & $>24$ & & 0.81 \\
\hline 18. & APL17 & 21 & & 0.74 \\
\hline 19. & APL18 & 18 & & 0.82 \\
\hline 20. & APL19 & 7 & & 0.89 \\
\hline
\end{tabular}

Table 2: Drug release kinetics and mechanism of the lomustine loaded nanoparticles based on release exponent and correlation coefficient. 
a polymeric wall at a shorter distance from the primary nanodroplet center, associated with a decrease in the resultant nanoparticle size [16].

On the other hand, the combination of stirring by homogenization and nanoprecipitation method produced particles with smaller size, for both tested solvents. The modified nanoparticle-production method, thus achieved a synergistic effect of energy input during different phase mixing processes, just in that short time period when nanodroplets were formed, organic solvent diffused, and nanoparticles solidified.

High stirring rates seemed to enhance the mass transfer and rate of diffusion between the multiphase, which induced high homogeneous supersaturation and rapid nucleation, to produce smaller drug particles. This method has the advantage of higher product yield, lower operating temperature, and high powder crystallinity and stability.

During gradual decrease of reducing pressure from 180 to $40 \mathrm{mmHg}$, there was gradual decrease of dispersion volume and consequently, an increase of the viscosity of dispersed droplets. This affected the droplet size equilibrium, involving the processes of droplet coalescence and agglomeration, during the early step of the solvent removal. By reducing pressure instantaneously at $40 \mathrm{mmHg}$, increased solvent front kinetic energy caused a higher degree of the droplet dispersion in the aqueous phase. Therefore, the local concentration of the organic internal phase, in the external aqueous phase was decreased and the diffusion rate was higher, thus resulting in smaller particles.

Polydispersity indices of these lomustine nanoparticles prepared by this method were low, and showed little variability between different batches of particles, prepared under various formulation conditions. All samples produced by shear forces, generated by stirring rates greater than $10,000 \mathrm{rpm}$ led to a PDI of $0.066-0.3$, which indicated reasonable size homogeneity of nanoparticles.

\section{Encapsulation efficiency}

Encapsulation efficiency increases with the increasing polymer concentration in acetone. The reason is that a high viscosity holds back the shear forces of solution, and avoids the leak of lomustine [19].

Lipophilicity of lomustine resulted in increased entrapment efficiency with increasing concentration, as lomustine was dispersed into the polymeric matrix of nanoparticles, and precipitated with polymer when put into the aqueous phase containing PVA, owing to its limited solubility. The speed of nanoparticle solidification by nanoprecipitation method enables the drug to be entrapped rapidly, thus preventing its diffusion into the outer phase [20].

Michailova et al. [21], observed that drug to polymer feed weight ratio influences the entrapment efficiency, irrespective of the solvent used of cononsolvent system. Entrapment efficiency was increased above $90 \%$, with drug to polymer ratio of $0.75: 1$ and $1: 1$. They explained that this was due to the sufficient amount of drug in the system, that promoted the formation of the micellar structure during the process of incorporation. Our study showed contrast results to this, as when we increased drug ratio by keeping ratio of polymer constant, entrapment efficiency was decreased. Instead, nonsolvent ethanol addition to the acetone increased the entrapment efficiency, and further increasing concentration of ethanol increased encapsulation efficiency, which might be due to quick diffusion, and dispersion of organic phase into aqueous phase, resulting in precipitation of PLA around emulsion droplets encapsulating the drug.

With the increase in the internal aqueous phase volume (amount of PVA solution), entrapment efficiency increased, which is similar to some extent with the observation of Jain et al. [22], who prepared nanoparticles by double emulsion solvent evaporation method, and showed that with the increase in the aqueous phase volume entrapment, efficiency increased up to an extent. Good emulsification could be one of the reasons for such behaviour.

Since the emulsion stabilizer PVA remains at the interface during solvent evaporation, there was a possibility of adsorption of the stabilizers as well as drug, which might have resulted in increased adsorption of drug, and increased entrapment efficiency with increased aqueous volume, and also with increased PVA concentration. This is in aggrement with findings of Freitas and Marchetti [23].

With increased homogenization speed and time, there was more entrapment of drug within the matrix of increased surface area particulates.

\section{In-vitro drug release}

The release of drug from polymeric nanoparticles was studied by the dialysis-bag diffusion technique. This is a method commonly used to study the release of drugs from colloidal suspensions $[20,24]$

Among the experimental method available for determining in vitro the release profiles from colloidal suspension, this method is the most suitable in order to separate rapidly and completely, nanoparticles from the release medium [25].

The release rate of the drug and its appearance in the outer dissolution medium is governed by the partition coefficient of the drug, between the polymer and the aqueous environment, and by the diffusion of the drug across the membrane as well [26].

The release pattern appeared monophasic. These findings indicate that the most part of the drug was only adsorbed or close to the surface of the nanoparticles, and not entrapped in the polymeric core [26] Nanoparticles may take shape before the precipitation of the drug then, the drug precipitation occurs onto the nanoparticle surface. This pattern of dissolution rate of lomustine embedded in the nanoparticles, might be an outcome of the nanoprecipitation procedure during nanoparticle formulation.

The drug release rate was increased with increasing concentration of stabilizer PVA, amount of PVA solution, lower concentration of polymer PLA, nonsolvent ethanol amount and homogenization speed and time. This increase of dissolution rate of lomustine embedded in the nanoparticles, might be the outcome of this preparation procedure during nanoparticle formulation. The nanoparticle size was also associated with these changes in drug-release kinetics. The smaller sized nanoparticles exhibited higher drug-release rates with corresponding increase in the total nanoparticle surface, resulting in a larger drug fraction exposed to the leaching medium, and shorter average diffusion path of the matrix-entrapped drug molecules [27]. Also, the incorporation of water soluble stabilizer PVA in the aqueous phase, and subsequently in the nanoparticle polymeric matrix facilitated the release of lomustine, with an accompanying slight increase of the carrier size and the more porous structure of the polymeric matrix.

The drug release rate was slowly observed with increased aqueous phase volume. This is in agreement with findings of Muthu and Singh [20], with increase in initial drug release rate. They explained that this resulted from a higher drug fraction extracted from the parent nanoparticles, by the acetone-rich external medium formed following the phase mixing. The drug escaping from the nanoparticles, subsequently readsorbs to the nanoparticle surface, after the evaporation of the organic solvents. 
The release pattern was found best fitted in zero order release kinetic model, with larger values of $\mathrm{r}^{2}$ near to unity. Zero order kinetics and Non-Fickian (anomalous) drug release from these nanoparticles, refers to a combination of diffusion and dissolution mechanism, which might be attributed to the modified nanoprecipitation method.

\section{Anticancer activity}

The anticancer activity, which was found of lomustine- loaded PLA nanoparticles could be ascribed to the controlled drug release feature of the nanoparticle formulation. Viability of the L132 cells treated with lomustine loaded PLA nanoparticles, was 5.6 times reduced more than that of the cells treated with lomustine at $100 \mu \mathrm{g} / \mathrm{ml}$ concentration, which means that the lomustine- loaded PLA nanoparticles are 5.6 times more effective than lomustine for L132 cell treatment. Furthermore, the nanoparticles formulation of lomustine actually should have been much more effective than lomustine, if the sustainable drug release feature for the nanoparticle formulation was considered, which was found to be $99.0 \%$ of the drug encapsulated in the PLA nanoparticles after $24 \mathrm{~h}$ release. We also did the viability experiment for L132 cells treated by lomustine or lomustine loaded PLA nanoparticles, at lomustine concentrations 20,50 and $75 \mu \mathrm{g} / \mathrm{ml}$. The corresponding results implied that lomustine loaded PLA nanoparticles were 1.6, 2 and 2.14 times more effective than lomustine treated cells at these concentrations, respectively. The advantage of lomustine nanoparticle formulation against lomustine drug seemed significant, also at such a low drug concentration. However, viability change at concentrations $100 \mu \mathrm{g} / \mathrm{ml}$ was more significant.

\section{Effect of solvent (acetone) and nonsolvent (ethanol) addition}

Generally ketones except ester isopropyl acetate are used as solvents. Among them, acetone is commonly used for nanoprecipitation. They are all able to dissolve PLA homopolymer and have a lower dielectric constant value than ethanol and methanol, which destines them rather for lipophilic drug encapsulation [28]. Actually, the dielectric constant of the solvent is certainly not responsible solely for an increase of nanoparticle size, since nanoprecipitation results from various phenomena that govern the diffusion of the solvent through the polymer, into the non-solvent. It is, therefore, expected that the choice of the solvent/non-solvent couple will affect the diffusion rate, and thus the final mean size more than individual solvent characteristics, like e.g., the dielectric constant, $\varepsilon$ or the Hildebrand solubility parameter, $\delta$. The affinity of the solvent for the non-solvent is of importance and, in this respect; the interaction parameter $\chi$ is certainly to be taken into consideration. This interaction is expressed as:

$$
\chi=\frac{V_{N S}}{R T}\left(\delta_{S}-\delta_{\mathrm{NS}}\right)^{2}
$$

Where, $V_{N S}$ is the molar volume of the nonsolvent $\left(58.5 \mathrm{~cm}^{3} / \mathrm{mol}\right.$ for ethanol).The higher the interaction parameter was, the larger the nanoparticles [28].

Reason of using alcohol as nonsolvent, was its low dielectric constant value. Indeed, lower the dielectric constant value; the more the non-solvent will dissolve hydrophobic compounds. Therefore, ethanol was the most suitable in this respect, since its dielectric constant value is 24.6, thus being far, from that of the value for water (80.1). Also, alcohols are not a concern in terms of toxicity, except for methanol. Indeed, they belong to class 3 according to the ICH solvent toxicity scale, whereas methanol appears amongst Class 2 solvents.

By the addition of ethanol nonsolvent, particle size was decreased. In this respect, it has been demonstrated that the rate of diffusion of the solvent into the non-solvent should certainly be considered; since the higher the rate of diffusion, the smaller the nanoparticles and the higher the yield of transformed polymer into nanoparticles [29].

\section{Effect of aqueous phase volume of PVA solution and PVA concentration}

The relationship between the amounts of polymer and stabilizer seemed to be related to the volume of the aqueous phase. The aqueous volume of $50 \mathrm{ml}$ and the stirring velocity of $19,000 \mathrm{rpm}$ were used, because we observed in the preliminary studies that these parameters affect particles size distribution. Thus, when the volume of the aqueous phase was increased, the particle size increased as well, possibly because it is much difficult to remove the organic phase from the formulation, and resulted in nanoparticles coalescence. Since the emulsion stabilizer remains at the oil/water $(\mathrm{O} / \mathrm{W})$ interface during solvent evaporation, there was a possibility of adsorption of the stabilizer, as well as drug which might have resulted in increased adsorption of drug, and increased entrapment efficiency with increased aqueous volume, and also with increased PVA concentration. This is in agreement with findings of Freitas and Marchetti [23].

The stabilizer amount that was in the continuous phase, could interfere with the nanoparticles. The higher concentrations of PVA in the continuous phase promoted the coagulation of the nanoparticles, resulting in increased particle size. This is in contrast to the findings of $\mathrm{Wu}$ et al. [10], that a high proportion of PVA could provide sufficient stabilization to the nanoparticles system, and reduces their particle size and size distribution.

Encapsulation efficiencies of lomustine increased with enhancing content of PVA. This finding is consistent with the reports from Wu et al. [10], Mu and Zhong [30], Sehra and Dhake [31]. The hydrophobic portion of PVA interpenetrated into the polymer chains during nanoprecipitation, and remained trapped to the polymeric matrix of the nanoparticles. Accordingly, the addition of PVA easily formed an interconnected network with drug-polymer, and thus elevated the encapsulation efficiencies of the drug.

The drug release rate was also observed with increased aqueous phase volume. This is in agreement with findings of Chorny et al. [16], with increase in initial drug release rate. This resulted from a higher drug fraction extracted from the parent nanoparticles, by the acetonerich external medium formed following the phase mixing. The drug escaping from the nanoparticles, subsequently readsorbed to the nanoparticle surface after the evaporation of the organic solvents.

\section{Conclusion}

The process optimization was aimed at producing monodispersed and nanosize particles with highest entrapment efficiency. We have identified several process variables that are crucial for preserving the anticancer activity of lomustine drug, during the production of nanoparticle-formulations. An optimised method was introduced to adjust the processing conditions, to give small size nanoparticles and to preserve the activity of lomustine. This modified nanoprecipitation method with PLA polymer, produced nanoparticles with increased anticancer activity at lower lomustine equivalent concentrations.

For getting stable nanoparticle solution, appropriate stabilizer PVA incorporation mode was incorporation into the aqueous phase, prior to phase mixing. Critical formulation and process parameters for preparation of lomustine nanoparticles by modified nanoprecipitation method, were $20: 1$ polymer:drug ratio, $50 \mathrm{ml}$ of $1 \% \mathrm{w} / \mathrm{v}$ PVA aqueous 
Citation: Mehrotra A, Pandit JK (2012) Critical Process Parameters Evaluation of Modified Nanoprecipitation Method on Lomustine Nanoparticles and Cytostatic Activity Study on L132 Human Cancer Cell Line. J Nanomed Nanotechol 3:149. doi:10.4172/2157-7439.1000149

phase volume and the homogenization of $19,000 \mathrm{rpm}$ for $5 \mathrm{~min}$, reducing pressure instantaneously at $40 \mathrm{mmHg}$ for organic solvent evaporation, nonsolvent ethanol addition in 20:3 ratio with respect to solvent acetone. Further, lomustine nanoparticles of batches APL and APL12 can be investigated for in vivo studies.

\section{Acknowledgements}

We acknowledge to Prof. Dr. A.K.Wahi (Director, College of Pharmacy, IFTM, Moradabad, U.P; India) for continuous support during study.

\section{References}

1. Helle A, Hirsjarvi S, Peltonen L, Hirvonen J, Wiedmer SK, et al. (2010) Novel, dynamic on-line analytical separation system for dissolution of drugs from poly (lactic acid) nanoparticles. J Pharm Biomed Anal 51: 125-130

2. Belbella A, Vauthier C, Fessi H, Devissaguet JP, Puisieux $F$ (1996) In vitro degradation of nanospheres from poly ( $D, L$-lactides) of different molecular weights and polydispersities. Int J Pharm 129: 95-102.

3. Makino K, Arakawa M, Kondo T (1985) Preparation and in vitro degradation properties of polylactide microcapsules. Chem Pharm Bull (Tokyo) 33: 11951201.

4. Fessi, H, Puisieux F, Devissaguet JP, Ammoury N, Benita S (1989) Nanocapsule formation by interfacial polymer deposition following solvent displacement. Int J Pharm 55: R1-R4.

5. Allemann E, Gurny R, Doelker E (1992) Preparation of aqueous polymeric nanodispersions by a reversible salting-out process: influence of process parameters on particle size. Int J Pharm 87: 247-253.

6. Niwa T, Takeuchi H, Hino T, Kunou N, Kawashima $Y$ (1994) In vitro drug release behaviour of $\mathrm{D}, \mathrm{L}$-lactide/glycolide copolymer (PLGA) nanospheres with nafarelin acetate prepared by a novel spontaneous emulsification solvent diffusion method. J Pharm Sci 83: 727-732.

7. Quintanar-Guerrero D, Allemann E, Fessi H, Doelker E (1998) Preparation techniques and mechanisms of formation of biodegradable nanoparticles from preformed polymers. Drug Dev Ind Pharm 24: 1113-1128.

8. Italia JL, Yahya MM, Singh D, Ravi Kumar MN (2009) Biodegradable nanoparticles improve oral bioavailability of amphotericin B and show reduced nephrotoxicity compared to intravenous Fungizone. Pharm Res 26: 1324-1331.

9. Yen FL, Wu TH, Lin LT, Cham TM, Lin CC (2009) Naringenin-loaded nanoparticles improve the physicochemical properties and the hepatoprotective effects of naringenin in orally-administered rats with $\mathrm{CCl}(4)$ - induced acute liver failure. Pharm Res 26: 893-902.

10. Wu TH, Yen FL, Lin LT, Tsai TR, Lin CC, et al. (2008) Preparation, physicochemical characterization, and antioxidant effects of quercetin nanoparticles. Int J Pharm 346: 160-168.

11. Keck CM, Muller RH (2006) Drug nanocrystals of poorly soluble drugs produced by high-pressure homogenization. Eur J Pharm Biopharm 62: 3-16.

12. Peltonen L, Koistinen P, Hirvonen J (2003) Preparation of nanoparticles by the nanoprecipitation of low molecular weight poly (I)lactide. AAPS PharmSciTech 13: $299-304$.

13. Hyvonen S, Peltonen L, Karjalainen M, Hirvonen J (2005) Effect of nanoprecipitation on the physicochemical properties of low molecular weight poly (L-lactic acid) nanoparticles loaded with salbutamol sulphate and beclomethasone dipropionate. Int J Pharm 295: 269-281.

14. Chacon M, Berges L, Molpeceres J, Aberturas MR, Guzman M (1996) Optimized preparation of poly $D, L$ (lactic-glycolic) microspheres and nanoparticles for oral administration. Int J Pharm 141: 81-91.

15. Molpeceres J, Guzman M, Aberturas MR, Chacon M, Berges L (1996) Application of central composite designs to the preparation of polycaprolactone nanoparticles by solvent displacement. J Pharm Sci 85: 206-213.

16. Chorny M, Fishbein I, Danenberg HD, Golomb G (2002) Lipophilic drug loaded nanospheres prepared by nanoprecipitation: effect of formulation variables on size, drug recovery and release kinetics. J Control Release 83: 389-400.

17. Govender T, Riley T, Ehtezazi T, Garnett MC, Stolnik S, et al. (2000) Defining the drug incorporation properties of PLA-PEG nanoparticles. Int J Pharm 199: 95-110.
18. Lamprecht A, Ubrich N, Yamamoto H, Schafer U, Takeuchi H, et al. (2001) Design of rolipram-loaded nanoparticles: comparison of two preparation methods. J Control Release 71: 297-306.

19. Gao H, Yang YW, Fan YG, Ma JB (2006) Conjugates of poly (DL-lactic acid) with ethylenediamino or diethylenetriamino bridged bis( $\beta$-cyclodextrin)s and their nanoparticles as protein delivery systems. J Control Release 112: $301-$ 311.

20. Muthu MS, Singh S (2008) Studies on biodegradable polymeric nanoparticles of risperidone: in vitro and in vivo evaluation. Nanomedicine (Lond) 3: 305-319.

21. Michailova V, Berlinova I, Iliev P, Ivanov L, Titeva S, et al. (2010) Nanoparticles formed from PNIPAM-g-PEO copolymers in the presence of indomethacin. In J Pharm 384: 154-164.

22. Jain AK, Goyal AK, Gupta PN, Khatri K, Mishra N, et al. (2009) Synthesis, characterization and evaluation of novel triblock copolymer based nanoparticles for vaccine delivery against hepatitis B. J Control Release 136: 161-169.

23. Freitas MN, Marchetti JM (2005) Nimesulide PLA microspheres as a potential sustained release system for the treatment of inflammatory diseases. Int $J$ Pharm 295: 201-211.

24. Lerouell-Le Verger $M$, Fluckiger L, Kim YI, Hoffman M, Maincent $P$ (1998) Preparation and characterization of nanoparticles containing an antihypertensive agent. Eur J Pharm Biopharm 46: 137-143.

25. Soppimath KS, Aminabhavi TM, Kulkarni AR, Rudziski WE (2001) Biodegradable polymeric nanoparticles as drug delivery devices. J Control Release 70: 1-20.

26. Leo E, Brina B, Forni F, Vandelli MA (2004) In vitro evaluation of PLA nanoparticles containing a lipophilic drug in water-soluble or insoluble form. Int J Pharm 278: 133-141.

27. Brigger I, Dubernet C, Couvreur P (2012) Nanoparticles in cancer therapy and diagnosis. Adv Drug Deliv Rev 54.

28. Bilati U, Allemann E, Doelker E (2005) Development of a nanoprecipitation method intended for the entrapment of hydrophilic drugs into nanoparticles. Eur J Pharm Sci 24: 67-75

29. Stainmesse S, Orecchioni AM, Nakache E (1992) Modelling of an original process to obtain biocompatible polymeric nanospheres. Proc Sixth Congr Int Technol Pharm 1: 89-97.

30. Mu X, Zhong Z (2006) Preparation and properties of poly(vinyl alcohol)stabilized liposomes. Int J Pharm 318: 55-61.

31. Sehra S, Dhake AS (2005) Formulation and evaluation of sustained release microspheres of poly-lactide-co-glycolide containing tamoxifen citrate. J Microencapsul 22: 521-528. 